\title{
Video Article \\ Laboratory Drop Towers for the Experimental Simulation of Dust-aggregate Collisions in the Early Solar System
}

\author{
Jürgen Blum ${ }^{1}$, Eike Beitz ${ }^{1}$, Mohtashim Bukhari ${ }^{1}$, Bastian Gundlach ${ }^{1}$, Jan-Hendrik Hagemann ${ }^{1}$, Daniel Heißelmann ${ }^{1}$, Stefan Kothe ${ }^{1}$, \\ Rainer Schräpler ${ }^{1}$, Ingo von Borstel ${ }^{1}$, René Weidling ${ }^{1}$ \\ ${ }^{1}$ Institut für Geophysik und extraterrestrische Physik, Technische Universität Braunschweig
}

Correspondence to: Jürgen Blum at j.blum@tu-bs.de

URL: https://www.jove.com/video/51541

DOI: doi: $10.3791 / 51541$

Keywords: Physics, Issue 88, astrophysics, planet formation, collisions, granular matter, high-speed imaging, microgravity drop tower

Date Published: 6/5/2014

Citation: Blum, J., Beitz, E., Bukhari, M., Gundlach, B., Hagemann, J.H., Heißelmann, D., Kothe, S., Schräpler, R., von Borstel, I., Weidling, R. Laboratory Drop Towers for the Experimental Simulation of Dust-aggregate Collisions in the Early Solar System. J. Vis. Exp. (88), e51541, doi:10.3791/51541 (2014).

\section{Abstract}

For the purpose of investigating the evolution of dust aggregates in the early Solar System, we developed two vacuum drop towers in which fragile dust aggregates with sizes up to $\sim 10 \mathrm{~cm}$ and porosities up to $70 \%$ can be collided. One of the drop towers is primarily used for very low impact speeds down to below $0.01 \mathrm{~m} / \mathrm{sec}$ and makes use of a double release mechanism. Collisions are recorded in stereo-view by two highspeed cameras, which fall along the glass vacuum tube in the center-of-mass frame of the two dust aggregates. The other free-fall tower makes use of an electromagnetic accelerator that is capable of gently accelerating dust aggregates to up to $5 \mathrm{~m} / \mathrm{sec}$. In combination with the release of another dust aggregate to free fall, collision speeds up to $\sim 10 \mathrm{~m} / \mathrm{sec}$ can be achieved. Here, two fixed high-speed cameras record the collision events. In both drop towers, the dust aggregates are in free fall during the collision so that they are weightless and match the conditions in the early Solar System.

\section{Video Link}

The video component of this article can be found at https://www.jove.com/video/51541/

\section{Introduction}

It is generally accepted that planet formation starts with the non-gravitational accumulation of microscopically small dust grains into larger dust aggregates (see review by Blum \& Wurm) ${ }^{1}$. The dust particles collide within their protoplanetary disks due to Brownian motion, relative drift motions, and turbulence of the nebular gas (see review by Johansen et al. $)^{2}$. If the collision velocities are sufficiently low, the dust particles stick together to form larger agglomerates. A wealth of laboratory measurements over the past years have led to a dust-aggregate collision model that predicts the outcome of a pair of dust aggregates with arbitrary masses and collision velocities ${ }^{3}$. The basic collisional results are sticking (in general for small aggregate masses and low collision velocities), bouncing, and fragmentation (for high impact speeds). However, the transitions between these phases are not sharp and there are other outcomes, like, e.g. mass transfer or erosion. Applying this model to a typical protoplanetary disk predicts the growth of $\mathrm{cm}$-sized dust aggregates within a few thousand years ${ }^{4}$. The presence of $\mathrm{cm}$-sized dust aggregates has been extensively investigated by astronomical observations over the past years and can now be considered as established (see review by Testi et al. $)^{5}$ so that we conclude that the principle mechanism by which the first macroscopic bodies in young planetary systems form has been identified.

However, the further growth to bodies of at least kilometer sizes is not so clear. For the terrestrial-planet region, two hypotheses are currently discussed (see also the recent reviews on this matter by Johansen et al. ${ }^{2}$ and Testi et al. ${ }^{5}$ ): (i) concentration of the cm-sized dust aggregates by, e.g. the streaming instability ${ }^{6}$ and subsequent gravitational collapse ${ }^{7,8}$ and (ii) growth of a few "lucky winners" to larger sizes with subsequent mass accretion by the mass-transfer process ${ }^{9,10,11}$. In both models, $\mathrm{cm}$-sized dust aggregates undergo an enormous number of mutual collisions at low to moderate velocities. It is unclear what the possible outcomes of these collisions (besides bouncing) are.

To improve the dust-aggregate collision model by Güttler et al. ${ }^{3}$ and to investigate in more detail the collisions among macroscopic dust aggregates in the relevant velocity regimes, we set up two drop towers in our laboratory, in which individual aggregate-aggregate collisions can be studied in great detail under vacuum and microgravity conditions. Both drop towers possess a free-fall height of $1.5 \mathrm{~m}$, which limits the observation time to $\sim 0.5 \mathrm{sec}$. Thus, we observe the collisions by high-speed cameras with megapixel format and up to 7,500 frames per second. For maximum contrast and high recording speeds, bright-field illumination is chosen. Lighting is thus provided by high-intensity LED panels and homogenized by diffuser screens. Thus, the high-speed cameras view the colliding dust aggregates as dark objects in front of an illuminated screen. To avoid flickering, the LEDs are DC powered.

To achieve low collision velocities, the two dust aggregates are placed above one another in a double release mechanism. Releasing the upper aggregate a time of $t$ before the lower one results in a relative velocity of $v=g t$, with $g=9.81 \mathrm{~m} / \mathrm{sec}^{2}$ being the gravitational acceleration of the Earth. The two high-speed cameras, which view the collision from two directions $90^{\circ}$ apart, are typically released in between the two 
dust aggregates (typically $t / 2$ after the upper particle). The cameras run in continuous-recording mode, which is terminated by the impact of the camera holders into sand buckets. The maximum frame rate in this operational mode is 1,000 images per second at megapixel resolution. With this setup, velocities down to below $0.01 \mathrm{~m} / \mathrm{sec}$ have been achieved. Due to limitations of the mechanical setup of the double release mechanism, the maximum relative collision speed is $\sim 3 \mathrm{~m} / \mathrm{sec}$. Collisions involving dust aggregates with up to $5 \mathrm{~cm}$ in size have been investigated in this drop tower. For higher collision velocities up to $\sim 10 \mathrm{~m} / \mathrm{sec}$, a second drop tower is used, which is equipped with an electromagnetic accelerator that is capable to smoothly accelerate dust aggregates up to $5 \mathrm{~m} / \mathrm{sec}$ in a vertical upward direction. The other dust aggregate is held by a double-wing trap-door release mechanism and can be released rotation-free into free fall at any given time. Here, it does not make sense to use free-falling cameras. We rather use two stationary high-speed cameras with up to 7,500 frames per second and megapixel resolution. Due to the larger diameter of this drop tower, dust aggregates up to (and possibly above) $10 \mathrm{~cm}$ in size can be used.

\section{Protocol}

CAUTION: Depending on the hazardousness of the used particles, which can be found in the corresponding Safety Data Sheets, mouth protection and safety gear must be worn by the person working with the dust. It is also recommended to use a suction system to keep the ambient air dust-free.

\section{Preparation of $\mathrm{cm}$-sized Dust Aggregate Samples}

1. Calculate the amount of required material by $m=\Phi \rho_{0} V$, where $m$ is the required mass, $\Phi$ is the desired volume filling factor (volume filling factor $=1$ - porosity), $\rho_{0}$ is the material density, and $V$ is the volume of the sample. $77 \mathrm{~g}$ of irregular silicate dust $\left(\rho_{0}=2.6 \mathrm{~g} / \mathrm{cm}^{3}\right)$ is required to achieve a sample porosity of $70 \%$ (volume filling factor $=0.3$ ) for a cylindrical sample of $5 \mathrm{~cm}$ diameter and height, respectively.

Note: Formation of terrestrial planets starts with coagulation of micrometer-sized dust grains - predominantly composed of silicates - into cmsized porous bodies. A well-studied and suitable laboratory analog material is $\mathrm{SiO}_{2}$, which is available as irregular-shaped powder with a size distribution ranging from 0.5 to $10 \mu \mathrm{m}$ as well as in the form of monodisperse spherical grains for better comparability to theoretical models (see Table 1 and Figure 1).

\begin{tabular}{|l|l|l|l|l|}
\hline $\mathrm{SiO}_{2}$-monomer grain type & Manufacturer & Particle diameter & Particle shape & Example figure \\
\hline Monodisperse & Micromod & $1.52 \pm 0.06 \mu \mathrm{m}$ & Spherical & Figure 1 (left) \\
\hline Polydisperse & Sigma-Aldrich & $0.1-10 \mu \mathrm{m}$ & Irregular & Figure 1 (right) \\
\hline
\end{tabular}

Table 1. Characteristics of the $\mathrm{SiO}_{2}$ particles used in the dust-aggregate collision experiments.

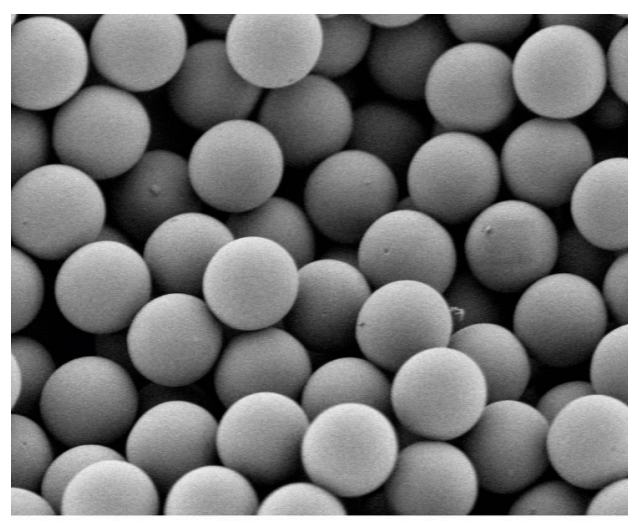

$2 \mu \mathrm{m}$

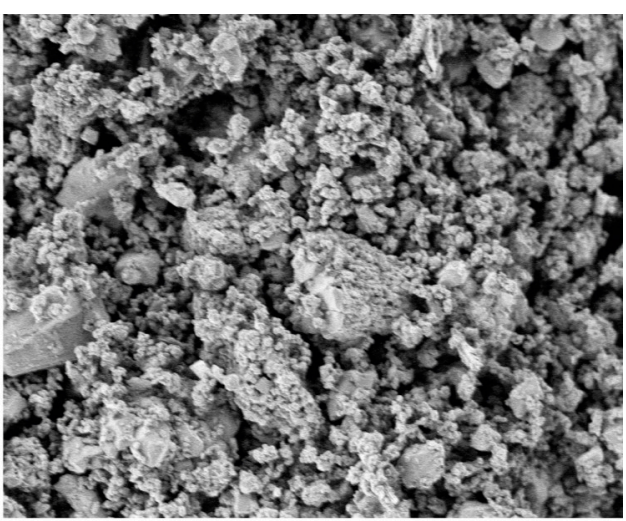

$5 \mu \mathrm{m}$

Figure 1. Electron-microscopy images of the monodisperse (left) and polydisperse (right) $\mathrm{SiO}_{2}$ particles used for the production of macroscopic dust aggregates. Please click here to view a larger version of this figure.

2. Take a container with micrometer-sized $\mathrm{SiO}_{2}$ particles (see Table 1) and pour its contents onto a sieve with a mesh size of $0.5 \mathrm{~mm}$. Sift a sufficient amount of material and fill the calculated mass into the mold. Compress the material in the mold by pushing in a piston by hand until the sample height is reached $(e . g .5 \mathrm{~cm})$. Turn around the mold on the piston, open the base plate and gently push the sample out. Note: Samples can be produced in several shapes (spherical and cylindrical), sizes (1 $\mathrm{mm}$ to $10 \mathrm{~cm}$ ) and porosities (60 to $85 \%$ ) (see Figure 3). Samples can then be used individually in the collision experiments or combined into clusters, which then collide with other aggregates or clusters. 


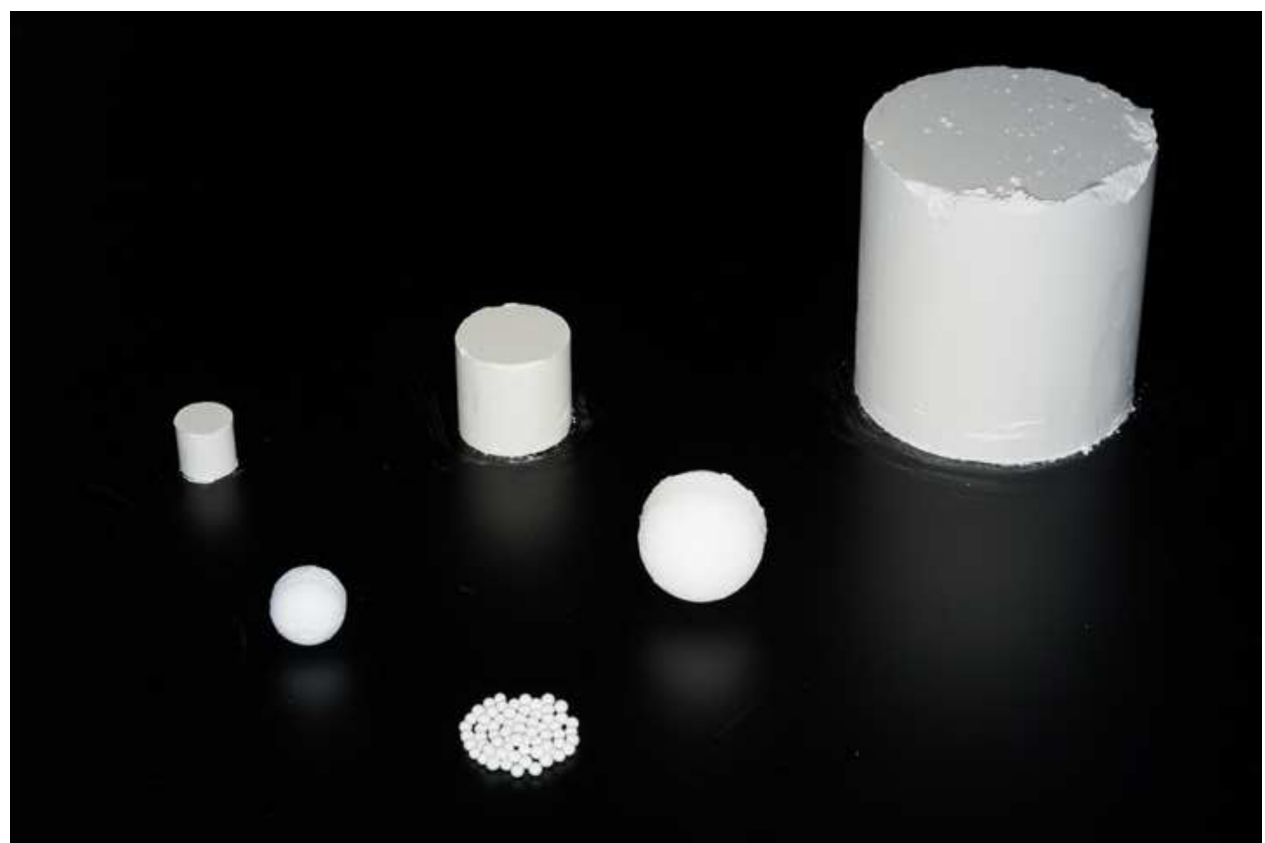

Figure 2. Photograph of the variation of dust-aggregate sample sizes and shapes. The following samples are shown: dust cylinders with $1 \mathrm{~cm}, 2 \mathrm{~cm}$, and $5 \mathrm{~cm}$ diameter (back row), dust spheres with $1 \mathrm{~cm}$ and $2 \mathrm{~cm}$ diameter (center row), and 2-3 mm-sized $\mathrm{Al}_{2} \mathrm{O}_{3}$ spheres (front). Please click here to view a larger version of this figure.

3. Characterize the samples with respect to porosity and homogeneity. If samples fall outside allowed margins, produce new samples.

1. In order to determine the porosity of the dust sample, determine its volume by measuring its dimensions and its mass by means of a precise balance.

2. Use X-ray tomography $(\mathrm{XRT})^{12}$ to gain information on the homogeneity and pore-size distribution of the produced sample. Note: For the $5 \mathrm{~cm}$-sized dust aggregates, we found deviations from the mean volume filling factor, i.e. the ratio of the mass density of the sample and the material density of the monomer-dust particles, of only about $1 \%$ within the bulk of the volume of the samples and a slightly larger increase of the volume filling factor by up to $8 \%$ towards the outer boundaries ${ }^{12}$. Figure 3 shows an XRT reconstruction of a cut through a cylindrical dust aggregate of $5 \mathrm{~cm}$ diameter and $5 \mathrm{~cm}$ height. We do not use XRT for each dust aggregate but examine the internal structure and homogeneity of random samples. 


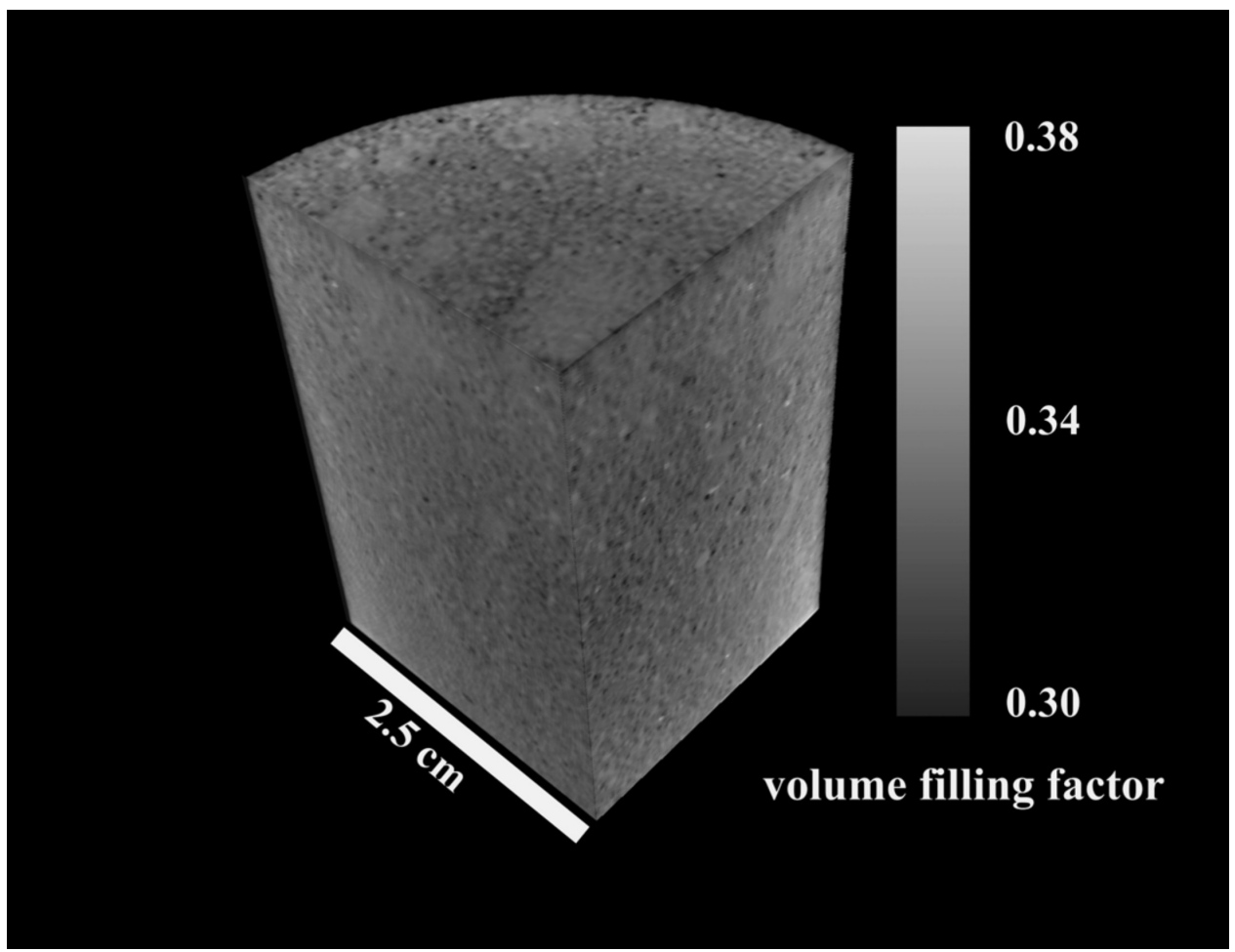

Figure 3. Reconstruction of the internal structure of a cylindrical dust-aggregate sample of $\mathbf{5 c m}$ height and $5 \mathrm{~cm}$ diameter after XRT analysis. The gray scale denotes the volume filling factor, which is the ratio of the mass density of the sample and the material density of the monomer-dust particles. From the XRT reconstruction, it is clearly visible that this high-porosity sample was assembled using mm-sized dust aggregates. Please click here to view a larger version of this figure.

\section{Principle of Drop Tower Setup}

1. Release mechanisms:

In the upper part of the drop tower two release mechanisms are attached one on top of the other. Each of them holds a sample and releases it into free fall. The time difference between the release of the upper and the lower particle determines the relative velocity of the collision. According to the morphology and the shape of the particles, appropriate release mechanisms are chosen. If a particle acceleration mechanism is used, only one release mechanism is required.

1. Particle-on-a-string release mechanism (spherical samples, upper particle):

This release mechanism consists of a linear solenoid magnet and a solid metal counter piece.

1. Attach the particle to be released to a string. Hold the string in place by clamping it between the solenoid magnet and the solid metal counter piece.

2. To release the particle, apply an electric current to the solenoid magnet (see Movie 1).

2. Trap-door release mechanism (spherical samples, lower particle):

This release mechanism consists of a rotary solenoid magnet to which a particle holder is attached.

1. Place the particle into a semi-spherical mold, which is rotated downward by a rotational solenoid when an electric current is applied (see Movie 1)

2. This mechanism can also be used for the release of particle clusters or aggregate clumps. In this latter case, mount two trapdoor release mechanisms above one another (see Movie 2).

3. Scissor-type double release mechanism (cylindrical samples):

This release mechanism consists of two pairs of rotary solenoid magnets to which a metal rod is attached. The two solenoid magnets of each release mechanism are placed such that the two metal rods are parallel.

1. Position the two samples onto the two parallel rods each.

2. Apply an electric current to the two rotational solenoids to release the particles into free fall. (see Movie 3).

4. Double-wing trap-door release mechanism (cylindrical samples, in combination with particle acceleration mechanism):

This release mechanism consists of two spring-loaded metal plates, which together form a v-shaped particle holder. The two metal plates are held in place by a metal rod, which is attached to a rotary solenoid magnet.

1. Place the cylindrical dust sample onto the closed trap-door.

2. Unlock the trap-door by applying an electric current to the solenoid magnet. To avoid bouncing-back of the doors, eddy-current brakes stop them (see Movie 4).

Note: It is important to release the particles into free fall without initial velocity and rotation. For this purpose several release mechanisms have been developed $(2.1 .1-2.1 .4)$. 
2. Particle acceleration mechanisms:

Accelerates the particles either by a pre-loaded spring or by an electro-magnetically driven linear stage. Both accelerators can be equipped with sample holders for differently shaped particles.

3. Control electronics:

Set the timer and release electronics to the appropriate values to achieve the desired collision velocity and to operate the camera in a centerof-mass frame.

Note: The timing of the particle release, particle acceleration and camera release is performed by a set of electronic timers, whose functionality is explained in Movie 5 .

\section{Performing Experiments}

1. Low-velocity collisions (small drop tower):

1. Load samples into the scissor-type double release mechanism and close vacuum glass tube.

2. Start evacuation and set timer parameters.

3. Attach cameras to their magnetic release units. Start continuous camera recording.

Note: Due to the high intensity of the LED bright-field illumination, a sufficiently short exposure time of the high-speed camera can be chosen so that the particle motion during the exposure is negligible. On top of that, the f-stop of the camera objective must be set to high enough values to extend the depth-of-focus over the whole diameter of the drop tower.

4. When the desired vacuum quality is reached, switch on lighting, press the start button and download the image sequences.

2. High-velocity collisions (large drop tower):

1. Load samples into the double-wing trap-door release mechanism and accelerator and close the vacuum glass tube.

2. Start evacuation and set timer parameters.

3. Start continuous camera recording. When the desired vacuum quality is reached, switch on lighting and press the start button. Download the image sequences.

Note: Due to the high intensity of the LED bright-field illumination, a sufficiently short exposure time of the high-speed camera can be chosen so that the particle motion during the exposure is negligible. On top of that, the f-stop of the camera objective must be set to high enough values to extend the depth-of-focus over the whole diameter of the drop tower.

\section{Example Experiments}

1. Load the samples carefully into the appropriate release mechanism.

1. Low-speed collisions (double release mechanism; $0.09 \mathrm{~m} / \mathrm{sec}$ ): $5 \mathrm{~cm}$ vs. $5 \mathrm{~cm}$, bouncing.

Load the samples into two scissor-type release mechanisms. To achieve collision velocities of $0.09 \mathrm{~m} / \mathrm{sec}$, place the particles $7 \mathrm{~mm}$ apart and set the time delay of the release mechanisms to 9 msec.

Note: At this impact velocity, the dust samples bounce off each other after the collision. The image sequence is captured by a freefalling high-speed camera (see Movie 6).

2. High-speed collisions (electromagnetic accelerator; $7.4 \mathrm{~m} / \mathrm{sec}$ ): $2 \mathrm{~cm}$ vs. $2 \mathrm{~cm}$, fragmentation.

Load one sample onto the double-wing trap-door release mechanism; place the other sample on the sample holder of the linear-stage accelerator.

Note: To achieve collision velocities of $7.4 \mathrm{~m} / \mathrm{sec}$, the lower dust aggregate is smoothly accelerated upward with $2 \mathrm{~g}$, while simultaneously the upper dust aggregate falls. At a relative velocity of $7.4 \mathrm{~m} / \mathrm{sec}$, the dust samples fragment (see Movie 7).

3. High-speed collision of small aggregates on big aggregates: $0.5 \mathrm{~cm}$ vs. $5 \mathrm{~cm}$, mass transfer.

Load the large sample onto a scissor-type release mechanism; place the smaller sample on the sample holder of the spring accelerator.

Note: To achieve collision velocities required for mass transfer, the lower dust aggregate is smoothly accelerated upward, while simultaneously the upper dust aggregate falls. At this relative velocity, the smaller sample fragments and transfers a small amount of mass onto the larger sample. As the camera falls along the upper (more massive) particle, the images taken by the high-speed camera give the impression of a large particle more or less at rest (see Movie 8), which is not true as seen from the outside of the drop tower.

2. Close the vacuum glass tube.

3. Carefully open the vacuum valve to the pumps to start slow evacuation and set the timer parameters to the required time difference for the desired collision velocity.

4. Attach cameras to their release units (if free-falling cameras are used). Start continuous camera recording and switch on lighting.

5. When the desired vacuum quality is reached, press the release button to initiate the timer sequence.

6. Download the image sequences recorded by the high-speed cameras to a computer.

\section{Data Analysis}

1. Chose an appropriate threshold gray value between the background and the objects' gray value. Create a binary image based on this threshold by setting pixels with gray values above the threshold to white (binary value 1) and pixels with lower gray values to black (binary value 0 ).

2. Determine the position of the particles' center of mass in each of the images. A good approximation to determine the center of mass for symmetrical particles is the center of the projected area. This is computed from binarized images.

3. Use the relative position of the objects' centers of mass and the time information from the camera images to calculate the relative velocity (see Movie 9). The slopes of the position curve are shown on the right-hand-side of Movie 9. 
1. In case of a rebounding collision, determine the relative velocities before and after contact. Calculate the coefficient of restitution, i.e. the ratio of the velocity after the collision and before. Plot the relative velocity against the coefficient of restitution. An example of this analysis is shown in Figure 4.

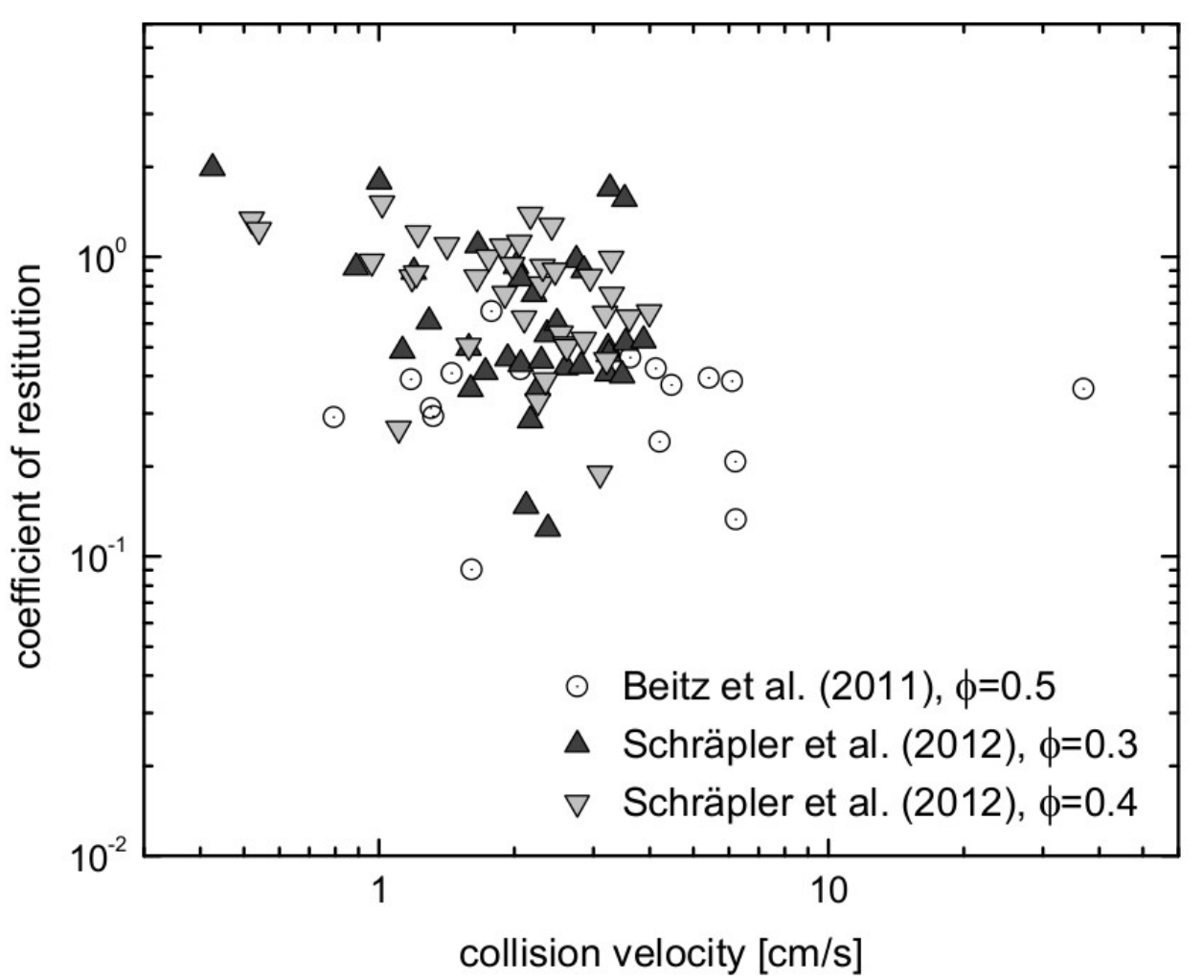

Figure 4. Example of the analysis of bouncing collisions. The coefficient of restitution, i.e. the ratio of the rebound velocity and the impact velocity, is plotted as a function of the collision velocity. Circles show data for spherical dust aggregates of $2 \mathrm{~cm} \mathrm{diameter}^{13}$ (see Figure 2), triangles denote collisions between cylindrical dust aggregates of $5 \mathrm{~cm}$ diameter and $5 \mathrm{~cm}$ height (see Figure 2) and two different volume filling factors of 0.3 and 0.4 , respectively ${ }^{12}$. The data show a trend of decreasing coefficient of restitution with increasing impact velocity. Please click here to view a larger version of this figure.

2. If one or both particles fragment, determine the sizes of as many of the largest fragments as possible by measuring their respective projected areas and assuming appropriate shapes.

1. If fragmentation of only one particle occurs, it usually transfers a certain amount of its mass to the surviving particle. Determine the amount of transferred mass by measuring the accreted volume, assuming an appropriate shape and porosity to quantify the masstransfer efficiency.

\section{Representative Results}

Using the well-characterized dust-aggregate samples described in the protocol (see Figures 1-3), any collision observed in one of the laboratory drop towers will yield scientifically valuable information on the outcomes of similar collisions in protoplanetary disks. We have so far systematically investigated the collision outcomes of $2 \mathrm{~cm}$ sized spherical dust aggregates (with volume filling factors of 0.5 ) in the velocity range between 0.008 and $2.02 \mathrm{~m} / \mathrm{sec}^{13}$ and of $5 \mathrm{~cm}$ sized cylindrical dust aggregates (with volume filling factors between 0.3 and 0.5 ) in the velocity range between 0.004 and $2 \mathrm{~m} / \mathrm{sec}^{12}$. We found bouncing between the dust aggregates as the dominating outcome for velocities below $\sim 0.4$ $\mathrm{m} / \mathrm{sec}$ for both types of dust aggregates (see Movie 6 for an example). In Figure 4, the coefficient of restitution of these bouncing collisions is shown. The circles denote the experiments with $2 \mathrm{~cm}$ sized spherical samples ${ }^{13}$ and the triangles represent results from collisions among $5 \mathrm{~cm}$ sized dust cylinders with two different packing densities ${ }^{12}$. Although the coefficients of restitution of individual experiments scatter widely, the average value of the coefficient of restitution decreases with increasing collision velocity.

Both dust aggregates typically fragment upon impact for velocities above $\sim 1 \mathrm{~m} / \mathrm{sec}$ (see Movie 7 for an example). For velocities between $\sim 0.4$ and $\sim 1 \mathrm{~m} / \mathrm{sec}$, fragmentation of only one of the two colliding dust aggregates can occur. In this case, the non-fragmenting dust aggregate gains a few percent of mass by mass transfer ${ }^{13}$. The above-mentioned velocity limits are not sharp but denote approximately where the boundaries between the different regimes lie ${ }^{2,11}$. For collisions between dust aggregates of different sizes and moderate velocities, impacts will generally not lead to the fragmentation of the larger of the two dust aggregates. On the opposite, the larger bodies increase their mass by transfer of part of the mass of the smaller impactors (see Movie 8).

For the cases, in which the two dust aggregates bounce off one another, the transfer from the translational kinetic energy before the collision (mind that the dust aggregates do not rotate before the collision) into translational kinetic energy, rotational kinetic energy, and other (dissipative) 
energy channels (e.g. compaction of the dust aggregates) can be determined. We found that for central collisions (in which the rotational energy can be neglected) the relative amount of dissipated energy strongly increases with increasing velocity and is higher for lower volume filling factors of the dust aggregates ${ }^{12}$. This behavior can be modeled by molecular-dynamics simulations ${ }^{12}$.

Movie 1. High-speed movie (played back in slow motion) of the particle-on-a-string (top) and trap-door release mechanism (bottom).

Movie 2. High-speed movie (played back in slow motion) of the double trap-door release mechanism. Both samples are clumps of $\mathrm{Al}_{2} \mathrm{O}_{3}$ particles of $2 \mathrm{~mm}$ diameter, which remain confined during free fall due to the extremely low disturbance during release.

Movie 3. High-speed movie (played back in slow motion) of the scissor-type double release mechanism.

Movie 4. High-speed movie (played back in slow motion) of the double-wing trap-door release mechanism.

Movie 5. Animation of the timer electronics switching the upper and lower release mechanism as well as the camera release to free fall.

Movie 6. High-speed movie (played back in slow motion) of a bouncing collision between two $5 \mathrm{~cm}$-sized dust-aggregate cylinders. The two dust aggregates are released by the scissor-type double release mechanism and collide with $0.09 \mathrm{~m} / \mathrm{sec}$ velocity.

Movie 7. High-speed movie (played back in slow motion) of two $2 \mathrm{~cm}$-sized cylindrical dust aggregates colliding at a relative velocity of $7.4 \mathrm{~m} /$ sec. Both aggregates fragment completely.

Movie 8. High-speed movie (played back in slow motion) of a $5 \mathrm{~mm}$-sized dust aggregate impacting a $5 \mathrm{~cm}$-sized cylindrical solid target. As the impact velocity of $4.3 \mathrm{~m} / \mathrm{sec}$ is above the fragmentation speed of the small dust aggregate, this breaks apart and transfers part of its mass to the target, which is clearly visible in the movie.

Movie 9. Determination of the particle trajectories by a semi-automatic particle-tracking algorithm. Here, the collision between two $2 \mathrm{~cm}$-sized spherical dust aggregates is shown.

\section{Discussion}

Due to the high mechanical precision, the failure rate of both drop towers is extremely low. This is of utmost importance, because the sample preparation may take up to several hours, depending on size, shape and porosity of the desired dust aggregates. It should be mentioned that large dust aggregates with very high porosities are extremely fragile and, thus, difficult to handle. It may occur that these dust aggregates break during extraction off the mold or transfer to the drop tower. In these cases, a new sample has to be prepared. Thus, it is important that the small drop tower allows reliable (and predictable) collision velocities down to $0.01 \mathrm{~m} / \mathrm{sec}^{11,13}$. The lowest impact speed so far achieved was $0.004 \mathrm{~m} /$ sec. These small impact velocities can only be reached for free particles in a microgravity environment. The laboratory drop tower is a cheap and versatile realization of such a microgravity facility.

Alternative methods to achieve low impact speeds make use of levitation techniques ${ }^{14,15}$ (e.g. by electromagnetic or aerodynamic levitation) but generally induce a force between the colliding particles, which has to be taken into account in the analysis of the collisions. Moreover, levitation often induces rotational motion ${ }^{14}$, which, if unwanted, does not allow rotation-free collisions but, on the other hand, might even allow realistic simulations of collisions among rotating particles. In case of aerodynamic levitation, air-cushion effects during the collision may induce unwanted conditions that do not match those in protoplanetary disks. However, levitation allows for unlimited observation time and repeatable experiments so that it has to be considered an alternative to the drop tower if the time limitation is essential. All our efforts so far have been concentrated on $\mathrm{SiO}_{2}$ as a representative of the silicates in the terrestrial-planet formation region of young Solar Systems. As most of the mass of protoplanetary disks is concentrated beyond the condensation point of water ice, it is essential to also study the collision behavior of aggregates consisting of $\mu \mathrm{m}$-sized $\mathrm{H}_{2} \mathrm{O}$-ice grains. We are currently setting up a cryo-vacuum drop tower for this purpose. It must be noted that the temperatures in such simulation experiments must be below $\sim 150 \mathrm{~K}$, which is the temperature of the so-called "snow line" in protoplanetary disks (the "snow line" divides the inner regions where water is in the vapor phase from the outer regions where it is found as solid water ice). We have shown that the formation of $\mu \mathrm{m}$-sized water-ice particles is feasible and that aggregates thereof can be produced ${ }^{16}$ so that we are optimistic to have first results on their collision behavior within the next 1-2 years.

\section{Disclosures}

We have nothing to disclose.

\section{Acknowledgements}

The authors thank the Deutsches Zentrum für Luft- und Raumfahrt (DLR), the Deutsche Forschungsgemeinschaft (DFG), and the TU Braunschweig for continual support of our laboratory activities. The low-velocity drop tower has been established under DLR grant 50WM0936, the high-velocity drop tower has been developed under DFG grant INST 186/959-1 as part of the CRC 963 "Astrophysical Flow Instabilities and Turbulence".

\section{References}

1. Blum, J., \& Wurm, G. The Growth Mechanisms of Macroscopic Bodies in Protoplanetary Disks. Annual Review of Astronomy and Astrophysics. 46, 21-56 (2008). 
2. Johansen, A., Blum, J., Tanaka, H., Ormel, C.W., Bizzarro, M., \& Rickman, H. The Multifaceted Planetesimal Formation Process. In: Protostars \& Planets VI. University of Arizona Space Science Series (2014).

3. Blum, J., Zsom, A., Ormel, C.W., \& Dullemond, C.P. The outcome of protoplanetary dust growth: pebbles, boulders, or planetesimals? I. Mapping the zoo of laboratory collision experiments. Astronomy and Astrophysics. 513, A56 (2010).

4. Zsom, A., Ormel, C.W., Güttler, C., Blum, J., \& Dullemond, C.P. The outcome of protoplanetary dust growth: pebbles, boulders, or planetesimals? II. Introducing the bouncing barrier. Astronomy and Astrophysics. 513, A57 (2010).

5. Testi, L. et al. Dust Evolution in Protoplanetary Disks In: Protostars \& Planets VI. University of Arizona Space Science Series (2014).

6. Youdin, A.N., \& Goodman, J. Streaming Instabilities in Protoplanetary Disks. The Astrophysical Journal. 620, 459-469 (2005).

7. Johansen, A., Youdin, A.N., \& Klahr, H. Particle Clumping and Planetesimal Formation Depend Strongly on Metallicity. The Astrophysical Journal Letters. 704, L75-L79 (2009).

8. Bai, X.N., \& Stone, J. M. Dynamics of Solids in the Midplane of Protoplanetary Disks: Implications for Planetesimal Formation. The Astrophysical Journal. 722, 1437-1459 (2010).

9. Windmark, F., Birnstiel, T., Güttler, C., Blum, J., \& Dullemond, C.P., Henning, Th. Planetesimal formation by sweep-up: How the bouncing barrier can be beneficial to growth. Astronomy and Astrophysics. 540, A73 (2012).

10. Windmark, F., Birnstiel, T., Ormel, C.W., \& Dullemond, C.P. Breaking through: The effects of a velocity distribution on barriers to dust growth Astronomy and Astrophysics. 544, L16 (2012).

11. Garaud, P., \& Meru, F. From Dust to Planetesimals: An Improved Model for Collisional Growth in Protoplanetary Disks. The Astrophysical Journal. 764, 146 (2013).

12. Schräpler, R., Blum, J., Seizinger, A., \& Kley, W. The physics of protoplanetesimal dust agglomerates. VII. The low-velocity collision behavior of large dust agglomerates. The Astrophysical Journal. 758, 35 (2012).

13. Beitz, E., Güttler, C., Blum, J., Meisner, T., Teiser, J., \& Wurm, G. Low-velocity collisions of centimeter-sized dust aggregates. The Astrophysical Journal. 736, 34 (2011).

14. Beitz, E., Blum, J., Mathieu, R., Pack, A., \& Hezel, D.C. Experimental investigation of the nebular formation of chondrule rims and the formation of chondrite parent bodies. Geochimica et Cosmochimica Acta. 116, 41-51 (2013).

15. Jankowski, T. et al. Crossing barriers in planetesimal formation: The growth of mm-dust aggregates with large constituent grains. Astronomy and Astrophysics 542, A80 (2012).

16. Gundlach, B., Kilias, S., Beitz, E., \& Blum, J. Micrometer-sized ice particles for planetary-science experiments - I. Preparation, critical rolling friction force, and specific surface energy. Icarus. 214, 717-723 (2011). 